\title{
Aspectos relacionados com a positividade para a esquistossomose: estudo transversal em área de baixa prevalência em Alagoas, 2020* \\ $10.1590 / 51679-49742021000200005$
}

\author{
Aspects related to positivity for schistosomiasis: a cross-sectional study in a low prevalence \\ area in Alagoas, Brazil, 2020
}

\section{Aspectos relacionados con la positividad para la esquistosomiasis: un estudio transversal en un área de baja prevalencia en Alagoas, Brasil, 2020}

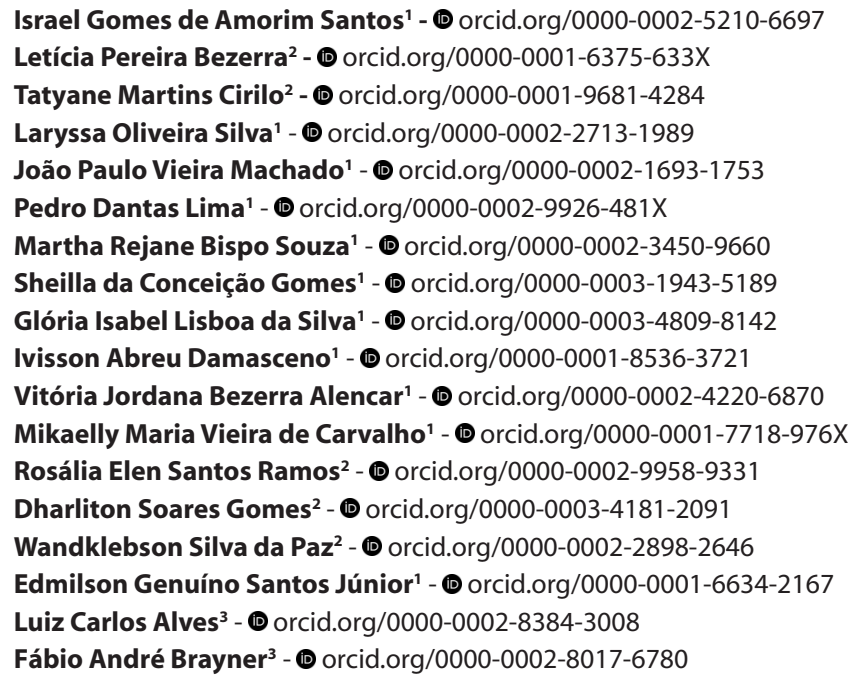

\section{Resumo}

Objetivo: Analisar aspectos relacionados com a positividade para esquistossomose em área de baixa prevalência, no Brasil. Métodos: Estudo transversal, realizado no primeiro semestre de 2020, quando foram analisadas a proporção de positividade, em função do número de lâminas de Kato-Katz, o desempenho diagnóstico do teste e a estimação da positividade a partir dos dados do Sistema de Informação do Programa de Vigilância e Controle da Esquistossomose (SISPCE). Resultados: Foram analisadas 2.088 lâminas de 348 indivíduos, sendo a proporção de positividade de 11,8\%, 26,7\% e 31,0\% para 1, 4 e 6 lâminas analisadas, respectivamente. Houve concordância excelente (índice Kappa =0,91) na comparação entre as leituras de 4 e 6 lâminas. Foi estimada subnotificação de 2,1 vezes nos dados do SISPCE. Conclusão: Ampliar o número de lâminas aumentou a positividade do Kato-Katz, o que pode contribuir para maximizar o controle da doença enquanto problema de Saúde Pública.

Palavras-chave: Doenças Negligenciadas; Estudos Transversais; Sensibilidade e Especificidade; Saúde Pública; Esquistossomose Mansônica; Doenças Parasitárias.

\footnotetext{
*Artigo derivado da tese de doutorado, intitulada 'Epidemiologia da esquistossomose mansoni em uma área de baixa prevalência no estado de Alagoas', a ser apresentada por Israel Gomes de Amorim Santos junto ao Programa de Pós-Graduação em Biociências e Biotecnologia em Saúde, do curso de Biociências e Biotecnologia em Saúde do Instituto Aggeu Magalhães, Fundação Instituto Oswaldo Cruz, Pernambuco. O estudo recebeu apoio financeiro da Fundação de Amparo à Pesquisa do Estado de Alagoas (Fapeal) e Ministério da Saúde: Processo n 60030.000857/2016.
}

Endereço para correspondência:

Israel Gomes de Amorim Santos - Estrada BR 316, Km 87,5, Bebedouro, Santana do Ipanema, AL, Brasil. CEP: $57500-000$

E-mail: israel.santos@uneal.edu.br 


\section{Introdução}

As esquistossomoses são doenças provocadas por vermes trematódeos do gênero Schistosoma. Essa morbidade está presente em mais de 78 países, dos quais 52 evidenciam necessidade de campanhas de tratamento em larga escala. ${ }^{1}$ A espécie de parasito presente no Brasil é o Schistosoma mansoni, que chegou ao país com o tráfico de pessoas escravizadas durante o período de colonização, inicialmente em estados da região Nordeste. Com o fim da mão de obra de pessoas escravizadas, o parasito e a doença foram levados para outras regiões, devido ao êxodo nordestino, tornando sua presença de grande relevância e magnitude para a Saúde Pública brasileira até os dias de hoje. ${ }^{2,3}$

\section{0 método de Kato-Kratz mostra-se efetivo no diagnóstico da infecção pelo S. mansoni apenas em áreas de alta prevalência, um cenário que não corresponde ao da maioria dos países endêmicos para a parasitose.}

Em 2019, a Secretaria de Vigilância em Saúde, do Ministério da Saúde, estimou 1,5 milhão de pessoas infectadas pelo $S$. mansoni no Brasil. 0 agravo está presente, de forma endêmica, em nove estados, persistindo como foco em outras nove Unidades da Federação (UFs) onde se observa a manutenção de suas características epidemiológicas. ${ }^{3.5}$ Diante da relevância da endemia, as autoridades de saúde lançaram mão de várias medidas de controle dos moluscos transmissores do $S$. mansoni e erradicação da doença no país.

0 exame parasitológico de fezes é o padrão ouro para o diagnóstico da infecção e controle da morbidade, desde sua otimização na década de 1970, com a criação do método quantitativo de Kato-Katz. ${ }^{6}$ Este é o método atualmente recomendado pela Organização Mundial da Saúde para os inquéritos coproparasitológicos nos países endêmicos. ${ }^{7}$ Entretanto, nos dias de hoje, o método de Kato-Kratz mostra-se efetivo no diagnóstico da infecção pelo $S$. mansoni apenas em áreas de alta prevalência, um cenário que não corresponde ao da maioria dos países endêmicos para a parasitose. Outros métodos, como os sorológicos e os imunocromatográficos baseados na detecção de antígenos do verme liberados na urina do indivíduo, são promissores, muito embora, em termos de sensibilidade e especificidade, insuficientes para substituir o método preconizado pelas autoridades de saúde nacionais e internacionais. ${ }^{8-10}$ Isto, a despeito de já haver indicação do emprego desses métodos em conjunto. ${ }^{11}$

Uma vez que (i) os indicadores epidemiológicos, provenientes das atividades do Programa de Controle da Esquistossomose (PCE), fundamentam a formulação de diretrizes de controle e erradicação da esquistossomose mansoni no Brasil, (ii) o método Kato-Katz não é sensível à detecção do $S$. mansoni em áreas de baixa prevalência e (iii) os métodos diagnósticos alternativos ainda não podem ser usados na rotina de equipes municipais, é imperativo que as diretrizes de diagnóstico e logística, atualmente empregadas no PCE, possam ser otimizadas. Dessa forma, poder-se-á conhecer a real situação epidemiológica da doença, contribuindo com a construção de políticas públicas de saúde mais efetivas no combate à endemia.

0 objetivo do presente estudo foi analisar aspectos relacionados com a positividade da esquistossomose em uma área de baixa prevalência da doença no Brasil.

\section{Métodos}

Realizou-se um estudo transversal no município de Lagoa da Canoa, Alagoas, nos meses de março e abril de 2020. Seus resultados foram comparados com dados reportados no Sistema de Informação do PCE para 0 período de 2007 a 2016.

0 município pertence à $7^{\mathrm{a}}$ Região de Saúde do estado (7/10), apresenta índice de desenvolvimento humano de 0,552 e índice de vulnerabilidade social de 0,490, ambos referentes ao ano de 2010. ${ }^{12}$ Lagoa da Canoa conta com unidades básicas de saúde (UBS), ambulatório especializado, postos de saúde, unidade mista e centro de atenção psicossocial. Em relação às doenças infecciosas e parasitárias, o município é endêmico para doença de Chagas, esquistossomose e leishmaniose tegumentar americana; ademais, é área de vigilância para leishmaniose visceral e peste. $^{13}$

A amostra do estudo foi definida considerando-se $3,0 \%$ de erro aceitável e $8,1 \%$ de prevalência, obtida pela média de dez anos (2007-2016) de registros no Sistema de Informação do Programa de Vigilância e Controle da Esquistossomose (SISPCE). A população local é estimada em 18.250 habitantes. ${ }^{12}$ A partir dessas referências, calculou-se uma amostra de 311 indivíduos, corrigida para 373 como compensação de possíveis perdas. 
No processo de amostragem, os indivíduos foram divididos proporcionalmente, de acordo com a zona de residência: aproximadamente, metade da população do município vive na zona urbana. Além disso, levou-se em consideração a população atendida por cada unidade da Estratégia Saúde da Família. Com a ajuda dos agentes comunitários de saúde (ACS), as residências foram selecionadas aleatoriamente, respeitando-se a distância de 50 a 100 metros entre elas, desde a primeira residência selecionada e assim por diante.

Foram elegíveis para o estudo indivíduos a partir de 5 anos de idade, sem histórico de uso de praziquantel nos seis meses anteriores à pesquisa. A informação sobre o uso ou não desse medicamento anti-helmítico e/ou antiparasitário foi autorreferida pelos participantes e/ ou seus responsáveis.

As variáveis do estudo foram trabalhadas conforme a natureza dos dados:

a) Dados primários

- Proporção de positividade da infecção pelo $S$. mansoni em função do número de lâminas de Kato-Katz analisadas.

- Proporção de positividade da infecção pelo $S$. mansoni por localidade do município.

- Intensidade da infecção, em função da leitura de uma a seis lâminas analisadas (leve [1 a 99 OPG]; moderada [100 a 399 OPG]; pesada [mais de 400 OPG]).

b) Dados secundários

- Proporção média de positividade da infecção pelo S. mansoni para cada localidade do município, no período de 2010 a 2016.

Cada participante foi informado, verbalmente, dos objetivos do estudo e do procedimento correto para a coleta do material fecal. De cada um deles foram coletadas três amostras fecais, em diferentes dias. Essas amostras foram recolhidas na casa de cada participante e armazenadas em caixa de isopor contendo gelo, para transporte seguro ao Laboratório de Parasitologia Humana e Malacologia da Universidade Estadual de Alagoas. Foram realizadas ao menos quatro visitas para a coleta do material, tendo sido excluídos do estudo os indivíduos que não fizeram a entrega de todas as amostras de fezes.

Analisou-se o material pelo método de Kato-Katz, sendo duas lâminas por amostra. Dois analistas treinados fizeram a leitura das lâminas. Quando não havia concordância em 30\% das leituras, quanto à presença/ ausência de ovos e/ou número de ovos contados, um terceiro analista, igualmente treinado no diagnóstico parasitológico da esquistossomose mansoni, realizava a leitura das lâminas. ${ }^{14}$

Foi considerado positivo para a infecção o indivíduo que apresentasse pelo menos um ovo do $S$. mansoni no material fecal analisado. 0 número de ovos por grama de fezes (OPG) resultou da multiplicação do número de ovos contados pelo fator de multiplicação 24 , conforme o protocolo do método de Kato-Katz. Para a apresentação final do $\mathrm{OPG}$, levou-se em consideração o número de lâminas lidas e a média aritmética dos ovos contados em todas as lâminas analisadas para cada indivíduo.

Os dados secundários utilizados no estudo foram coletados no SISPCE e referem-se ao período compreendido entre 2010 e 2016.

A proporção de positividade foi verificada sobre o número de lâminas positivas, considerando-se, isoladamente, a leitura de uma e de duas lâminas de cada amostra de fezes coletada. A proporção de casos positivos pela análise de uma lâmina de uma amostra foi comparada com a proporção de casos positivos pela análise de duas lâminas da mesma amostra, por meio do teste qui-quadrado de independência, sendo considerados significativos p-valores $<0,05$. Essa análise foi realizada para cada uma das três amostras de fezes coletadas, separadamente.

Foram estabelecidas a copositividade e a copositividade para a leitura de uma, duas e quatro lâminas de Kato-Katz, contra a leitura de seis lâminas, sendo esta última considerada o procedimento sob análise em relação à primeira leitura. A copositividade foi determinada pela razão entre o número de verdadeiros positivos e o número de lâminas diagnosticadas como positivas; $\mathrm{e}$ a conegatividade, como a razão entre os verdadeiros negativos e 0 número de lâminas determinadas como negativas. A concordância entre a leitura de uma, duas e quatro lâminas e a leitura de seis lâminas foi determinada pelo índice Kappa. ${ }^{15}$ Este índice foi estratificado nas seguintes categorias: nenhuma concordância (menor que 0,01 ); concordância ruim (entre 0,01 e 0,20 ); fraca (entre 0,21 e 0,40 ); moderada (entre 0,41 e 0,60 ); boa (entre 0,61 e 0,80 ); e concordância excelente (maior que 0,81 ). Para a comparação das proporções de lâminas positivas em função do número de lâminas lidas, foi utilizado o teste de McNemar, sendo os p-valores determinados pelo teste qui-quadrado.

Também foi comparada a positividade das leituras de uma, duas e quatro lâminas com a leitura de seis 
lâminas de Kato-Katz, determinando-se a razão entre a positividade resultante da leitura de seis lâminas $\mathrm{e}$ a positividade encontrada na leitura de cada um dos demais conjuntos de lâminas.

As proporções médias de positividade reportadas no SISPCE, para os anos de 2010 a 2016, foram comparadas com as proporções de positividade provenientes da leitura de uma a seis lâminas do material coletado nesse estudo.

Para a análise da estimação de positividade por localidades do município, foi criado um fator multiplicador baseado na diferença entre as positividades observadas no estudo de campo e nos dados disponibilizados no SISPCE. 0 cálculo da diferença entre as positividades foi realizado aplicando-se a seguinte fórmula:

$$
D p=p C-p P
$$

Na fórmula $D p$ corresponde à diferença nas positividades, $p C$ à positividade do estudo de campo e $p P$ à positividade dos dados do SISPCE. Considerando-se que, no estudo de campo, a proporção de positividade observada comportou-se em função do número de lâminas lidas - nesse caso, seis lâminas-, o fator de multiplicação resultou do somatório das razões das diferenças obtidas com a aplicação da fórmula (1), da seguinte forma:

$$
\text { Fator }=\sum\left(\frac{(D p) x \ldots(D p) n}{6}\right) / n(D p)
$$

O fator de multiplicação obtido com a fórmula (2) foi utilizado para uma estimação empírica da positividade da esquistossomose mansoni nas diversas localidades trabalhadas pela equipe local do PCE, utilizando-se para isso a positividade média no período de 2010 a 2016, calculada sobre os dados do SISPCE.

0 projeto do estudo foi submetido ao Comitê de Ética em Pesquisa com Seres Humanos da Universidade Federal de Alagoas (CEP/UFAL) - Certificado de Apresentação para Apreciação Ética (CAAE) $n^{0}$ 58695716.1.0000.5013 - e aprovado: Parecer $n^{\circ}$ 3.827.540, emitido em 7 de fevereiro de 2020. Todos os participantes e/ou seus responsáveis assinaram (i) o Termo de Consentimento Livre e Esclarecido e/ou (ii) o Termo de Assentimento Livre e Esclarecido para crianças e adolescentes.

\section{Resultados}

Participaram do estudo 348 indivíduos. Cada um deles forneceu três amostras de material coprológico,

Tabela 1 - Positividade para a esquistossomose mansoni em função da leitura de uma ou duas lâminas de Kato-

\begin{tabular}{|c|c|c|c|c|c|c|c|}
\hline \multirow{2}{*}{ Amostras } & \multicolumn{2}{|c|}{ Lâmina 1 + Lâmina 2} & \multicolumn{2}{|c|}{ Lâmina 1} & \multicolumn{2}{|c|}{ Lâmina 2} & \multirow{2}{*}{$\mathrm{p}$-valor ${ }^{\mathrm{a}}$} \\
\hline & $\mathrm{n}$ & Positividade $^{b}$ & $\mathrm{n}$ & Positividade $^{b}$ & $\mathrm{n}$ & Positividade $^{b}$ & \\
\hline \multicolumn{8}{|l|}{ Amostra 1} \\
\hline \multicolumn{8}{|l|}{ Positivo } \\
\hline Sim & 64 & & 51 & & 45 & & \\
\hline Não & 284 & 18,4 & 297 & 14,6 & 303 & 12,9 & $p<0,001$ \\
\hline Total geral & 348 & & 348 & & 348 & & \\
\hline \multicolumn{8}{|l|}{ Amostra 2} \\
\hline \multicolumn{8}{|l|}{ Positivo } \\
\hline $\operatorname{Sim}$ & 69 & & 57 & & 41 & & \\
\hline Não & 279 & 19,8 & 291 & 16,4 & 307 & 11,8 & $p<0,001$ \\
\hline Total geral & 348 & & 348 & & 348 & & \\
\hline \multicolumn{8}{|l|}{ Amostra 3} \\
\hline \multicolumn{8}{|l|}{ Positivo } \\
\hline Sim & 68 & & 50 & & 43 & & \\
\hline Não & 280 & 19,5 & 298 & 14,4 & 305 & 12,3 & $p<0,001$ \\
\hline Total geral & 348 & & 348 & & 348 & & \\
\hline
\end{tabular}
Katz analisadas de indivíduos de uma área de baixa prevalência, Alagoas, 2020 
para análise pelo método de Kato-Katz. Ao todo, foram examinadas 2.088 lâminas. A proporção de positividade para a esquistossomose mansoni variou de acordo com a quantidade de lâminas analisadas. A leitura de duas lâminas de cada amostra resultou em uma positividade que variou de 18,4\% (64 indivíduos) a 19,8\% (69 indivíduos). Essa proporção foi maior que a encontrada pela análise de uma lâmina, cuja variação foi de 11,8\% (41 indivíduos) a 16,4\% (57 indivíduos), sendo as diferenças entre as proporções estatisticamente significativas (Tabela 1). Observou-se aumento na proporção de positividade para $26,7 \%$ e $31,0 \%$ quando foram analisadas, respectivamente, quatro e seis lâminas.

Quanto à intensidade da infecção, 97 indivíduos foram classificados com infecção leve e um indivíduo com infecção pesada, quando foram analisadas seis lâminas de Kato-Katz de três amostras desses indivíduos. Quando foram examinadas duas lâminas de uma amostra, 58 indivíduos apresentaram infecção leve e dois apresentaram infecção pesada. Em relação à média de ovos por grama de fezes, na classe de infecção leve, a maior média foi de 37,8 ovos; na classe de infecção moderada, foi de 229,8 ovos; e na classe de infecção pesada, de 684,0 ovos (Tabela 2).

Ao se analisarem os indicadores diagnósticos da leitura de uma a seis lâminas de Kato-Katz, verificou-se copositividade de $88,7 \%$ quando comparada a leitura de quatro lâminas com a de seis lâminas, e de 48,0\% quando comparada a leitura de seis lâminas com a de uma lâmina. Além disso, o teste de McNemar mostrou bom desempenho na análise de seis lâminas, frente à análise de uma e duas lâminas: resultados do teste de McNemar de 53,02 e 40,02 respectivamente; $\mathrm{p}<0,001$ para ambos os resultados. 0 índice Kappa mostrou concordância excelente ao se comparar a leitura de quatro com a de seis lâminas (Kappa: 0,91). Quanto à diferença nas proporções de positividade, a análise de seis lâminas apresentou proporção de $31,0 \%$, ou seja, $1,2,1,7 \mathrm{e} 2,1$ vezes maior que a positividade proveniente da leitura de quatro $(26,7 \%)$, duas $(18,4 \%)$ e uma lâmina (14,6\%), respectivamente (Tabela 3).

Das 12 localidades estudadas que tiveram atividades do PCE em anos anteriores, dez apresentaram proporções médias de positividade mais altas, neste estudo, comparadas àquelas reportadas no SISPCE (Tabela 4). Além disso, mesmo considerando-se apenas a leitura de uma lâmina, a positividade encontrada no estudo foi superior à reportada para a maioria das localidades.

0 fator empírico, para se estimar a real proporção de positividade da esquistossomose mansoni nas localidades estudadas, foi igual a 2,1. Antes da correção da positividade de várias localidades do município por esse fator, cinco localidades apresentavam proporção média de positividade variando de 0,9 a 5,0\%, 18 localidades apresentavam esse indicador entre 6,2 e 14,8\%, enquanto para dez localidades essa variação foi de 15,4 a 39,9\%. Após a aplicação do fator de correção, o município passou a ter duas localidades com proporção média de positividades de $1,9 \%$ e $4,4 \%$, seis localidades com 0 indicador variando de 6,4 a 14,0\%, e 25 localidades com variação de 16,8 a 84,0\% (Tabela 5).

\section{Tabela 2 - Intensidade da infecção esquistossomótica e média de ovos por grama de fezes em função do númerode lâminas lidas em uma área de baixa prevalência, Alagoas, 2020}

\begin{tabular}{|c|c|c|c|}
\hline \multirow{2}{*}{ Amostra } & \multicolumn{3}{|c|}{ Infecção esquistossomótica } \\
\hline & $\begin{array}{c}\text { Leve }^{\mathrm{a}} \\
\text { (média de ovos) }^{\mathrm{b}}\end{array}$ & $\begin{array}{c}\text { Moderada } \\
\text { (média de ovos) }^{\mathrm{b}}\end{array}$ & $\begin{array}{c}\text { Pesada }^{\mathrm{a}} \\
\text { (média de ovos) }\end{array}$ \\
\hline 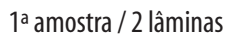 & $58(31,6)$ & $4(196,5)$ & $2(684,0)$ \\
\hline $2^{\mathrm{a}}$ amostra / 2 lâminas & $62(29,3)$ & $6(206,0)$ & $1(528,0)$ \\
\hline $3^{a}$ amostra / 2 lâminas & $58(37,8)$ & $10(229,8)$ & $1(420,0)$ \\
\hline 2 amostras / 4 lâminas & $86(30,2)$ & $6(209,0)$ & $1(552,0)$ \\
\hline 3 amostras / 6 lâminas & $97(32,3)$ & $9(193,8)$ & $1(406,0)$ \\
\hline
\end{tabular}

a) Número de indivíduos infectados de acordo com a intensidade da infecção pelo $S$. mansoni. Intensidade da infecção: resultado do número de ovos por grama de fezes, sendo classificada como leve (até 99 OPG), moderada (100 a 399 OPG) e pesada (a partir de 400 OPG); b) Média do número de ovos por grama de fezes. 
Tabela 3 - Desempenho diagnóstico da ampliação do número de lâminas lidas sobre a positividade da esquistossomose mansoni em uma área de baixa prevalência, Alagoas, 2020

\begin{tabular}{|c|c|c|c|c|c|c|c|}
\hline \multirow{2}{*}{$K^{\prime a}$} & \multicolumn{4}{|c|}{6 lâminas analisadas } & \multicolumn{2}{|c|}{ Positividade } & \multirow{2}{*}{$\begin{array}{l}\text { Relação entre } \\
\text { as positividades }\end{array}$} \\
\hline & Copositividade & Conegatividade & $\begin{array}{c}\text { McNemar } \\
\text { p-valor }\end{array}$ & Kappa $^{b}$ & Referênciac & 6 lâminas & \\
\hline 1 & $48,0 \%$ & $100,0 \%$ & $\begin{array}{c}53,02 \\
p<0,001\end{array}$ & 0,56 & $14,6 \%$ & $31,0 \%$ & 2,1 \\
\hline 2 & $60,0 \%$ & $100,0 \%$ & $\begin{array}{c}40,02 \\
p<0,001\end{array}$ & 0,67 & $18,4 \%$ & $31,0 \%$ & 1,7 \\
\hline 4 & $88,7 \%$ & $100,0 \%$ & $\begin{array}{c}1,00 \\
p>0,05\end{array}$ & 0,91 & $26,7 \%$ & $31,0 \%$ & 1,2 \\
\hline
\end{tabular}

a) KK:número de lâminas de Kato-Katz analisadas; b) Índice Kappa calculado; c) Representa a positividade resultante da leitura de 1,2 e 4 lâminas.

Tabela 4 - Comparação entre a positividade média obtida no estudo e a positividade média reportada no Sistema de Informação do Programa de Vigilância e Controle da Esquistossomose (SISPCE), localidades de Lagoa da Canoa, Alagoas, 2020

\begin{tabular}{|c|c|c|c|c|c|c|c|c|}
\hline \multirow{2}{*}{ Localidades } & \multirow{2}{*}{$\begin{array}{c}\text { Média de positividade (\%) } \\
\text { Dados do SISPCE } \\
2010-2016\end{array}$} & \multicolumn{7}{|c|}{$\begin{array}{c}\text { Média de positividade (\%) } \\
\text { Dados do estudo }{ }^{\mathrm{a}}\end{array}$} \\
\hline & & 1 & 2 & 3 & 4 & 5 & 6 & $7^{\mathrm{a}}$ \\
\hline Alto do Cruzeiro & 23,3 & $50^{b}$ & $50^{b}$ & 0 & 0 & 0 & $50^{b}$ & $50^{\mathrm{b}}$ \\
\hline Antunica de Baixo & 17,1 & 0 & $40^{b}$ & $20^{b}$ & $20^{b}$ & $20^{b}$ & 0 & $40^{\mathrm{b}}$ \\
\hline Antunica de Cima & 3,2 & $8^{b}$ & $8^{b}$ & $8^{b}$ & $8^{b}$ & $38^{b}$ & $15^{b}$ & $38^{\mathrm{b}}$ \\
\hline Barro Preto & 11,6 & $20^{b}$ & $30^{b}$ & 10 & 0 & $20^{b}$ & 0 & $50^{b}$ \\
\hline Cavaco & 11,4 & 0 & 0 & 10 & 0 & $30^{\mathrm{b}}$ & 10 & $40^{\mathrm{b}}$ \\
\hline Funil & 20,6 & 0 & 0 & 8 & 8 & 8 & 0 & 8 \\
\hline Lagoa da Pedra & 12,1 & $13^{b}$ & $13^{b}$ & $13^{b}$ & 0 & 0 & 0 & $25^{b}$ \\
\hline Lagoa do Mato & 13,4 & 9 & 9 & $27^{b}$ & $18^{b}$ & $18^{\mathrm{b}}$ & $18^{b}$ & $36^{b}$ \\
\hline Mata Limpa & 10,0 & 4 & 0 & 4 & 8 & 0 & 4 & $16^{b}$ \\
\hline Olho d'Água da Canoa & 15,4 & $28^{b}$ & $33^{b}$ & $22^{b}$ & $22^{b}$ & $17^{\mathrm{b}}$ & 11 & $44^{b}$ \\
\hline Pau d'Arco & 40,0 & 6 & 6 & 0 & 6 & 0 & 0 & 6 \\
\hline Zona Urbana & 9,4 & $22^{b}$ & $17^{b}$ & $27^{b}$ & $17^{\mathrm{b}}$ & $20^{b}$ & $20^{\mathrm{b}}$ & $42^{b}$ \\
\hline
\end{tabular}

a) A sequência numérica (1 e 2; 3 e 4; 5 e 6) representa o par de lâminas de cada amostra coletada. 0 número 7 representa a positividade média considerando-se a leitura das 6 lâminas como um único resultado; b) Positividade média observada no estudo maior do que a positividade média reportada no SISPCE para cada localidade do município. 
Tabela 5 - Positividade média da esquistossomose mansoni estimada, de acordo com um fator multiplicador resultante de inquérito de campo e de dados do Sistema de Informação do Programa de Vigilância e Controle da Esquistossomose (SISPCE), para localidades da cidade de Lagoa da Canoa, Alagoas, 20102016

\begin{tabular}{|c|c|c|}
\hline Localidade & $\begin{array}{l}\text { Positividade média } \\
\text { Registrada }^{\mathrm{a}}\end{array}$ & $\begin{array}{c}\text { Positividade média } \\
\text { Estimada }^{\mathrm{b}}\end{array}$ \\
\hline Alexandre & 6,3 & 13,2 \\
\hline Alto do Cruzeiro & 23,3 & 48,9 \\
\hline Antunica de Baixo & 17,1 & 35,9 \\
\hline Antunica de Cima & 3,2 & 6,7 \\
\hline Barro Preto & 11,6 & 24,3 \\
\hline Barro Vermelho & 0,9 & 1,9 \\
\hline Boa Vista I & 3,0 & 6,4 \\
\hline Campestrinho & 34,1 & 71,6 \\
\hline Capim & 11,1 & 23,2 \\
\hline Cavaco & 11,4 & 23,9 \\
\hline Chã do Pau d'Arco & 14,8 & 31,1 \\
\hline Folha Miúda de Baixo & 2,1 & 4,4 \\
\hline Funil & 20,6 & 43,3 \\
\hline Jenipapo & 8,5 & 17,8 \\
\hline Jurema & 9,7 & 20,3 \\
\hline Lagoa da Braúna & 6,2 & 13,1 \\
\hline Lagoa da Canoa & 9,4 & 19,8 \\
\hline Lagoa do Mato & 13,4 & 28,2 \\
\hline Lagoa da Pedra & 12,1 & 25,5 \\
\hline Lagoa das Varas & 10,7 & 22,5 \\
\hline Lagoa Grande & 8,0 & 16,8 \\
\hline Lagoa Queimada & 11,3 & 23,8 \\
\hline Mata Limpa & 10,1 & 21,1 \\
\hline Olho d'Água da Canoa & 15,4 & 32,4 \\
\hline Padre Cícero & 6,7 & 14,0 \\
\hline Pau d'Arco & 39,9 & 84,0 \\
\hline Pintada & 5,0 & 10,6 \\
\hline Riacho Grande & 20,0 & 42,0 \\
\hline Riacho Fundo & 11,3 & 23,7 \\
\hline Riacho Fundo de Cima & 20,0 & 42,0 \\
\hline Santa Clara & 26,1 & 54,8 \\
\hline São José III & 8,0 & 16,9 \\
\hline Sizília & 16,9 & 35,5 \\
\hline
\end{tabular}

a) Registrada no Sistema de Informação do Programa de Vigilância e Controle da esquistossomose (SISPCE); b) Estimada a partir do fator 2,1: fator empírico, construído a partir da diferença entre a positividade encontrada no estudo e aquelas reportadas para várias localidades do município de estudo. 


\section{Discussão}

Os resultados deste estudo mostraram que a ampliação no número de lâminas de Kato-Katz examinadas aumentou significativamente a positividade para o agravo, com maior concordância na mensuração da proporção de positividade à medida que houve a ampliação do número de lâminas. A aplicação de um fator empírico de estimação, por sua vez, sugere que a positividade nas diversas localidades do município está subestimada, fato relevante do ponto de vista epidemiológico, dentro de um contexto de eliminação do agravo em áreas de baixa prevalência para a esquistossomose mansoni.

Possíveis inconsistências nos dados obtidos do SISPCE, relacionadas com a metodologia empregada na análise da positividade para a infecção pelo $S$. mansoni e a alimentação do sistema, juntamente com o não ajuste dos dados do estudo, podem representar uma limitação para esta pesquisa, no que toca à comparação das positividades encontradas, apesar de haver-se escolhido um período longo (sete anos) para a coleta dos dados que compuseram a positividade média utilizada na análise, com vistas a uma melhor estabilidade dos dados. ${ }^{3}$ Ademais, possíveis erros de subestimação ou superestimação dos dados exigem cautela em sua interpretação, a partir de um fator empírico criado. Esta limitação pode ser minimizada com a validação de tal fator e sua utilização em diferentes cenários epidemiológicos do agravo.

A alta positividade encontrada no estudo, proveniente da ampliação do número de lâminas analisadas, mostra um cenário muito preocupante, seja por conta das diretrizes vigentes, seja diante das metas estabelecidas para o controle e erradicação da parasitose. Em 2012, foi lançado o plano integrado de ações estratégicas de eliminação de algumas doenças negligenciadas, incluindo a esquistossomose. A meta do plano era eliminar a esquistossomose enquanto problema de Saúde Pública no Brasil até o ano de 2015. Concomitantemente a esse plano, realizou-se um inquérito nacional de prevalência da esquistossomose e das geo-helmintoses, pelo qual se verificou que o Brasil não tinha atingido a meta de erradicação, e que o estado de Alagoas continuava a ser uma das UFs com as maiores prevalências para a morbidade por esquistossomose. ${ }^{16-18}$
É importante ressaltar a relevância da logística dos resultados observados no âmbito das ações municipais das equipes do PCE, tanto para 0 alcance das metas do plano integrado quanto para os resultados do inquérito nacional, pois ambos seguem as normatizações dos órgãos superiores de saúde do Brasil. A leitura de uma ou duas lâminas de Kato-Katz por indivíduo não é mais efetiva no sentido de estimar a prevalência da esquistossomose em áreas de baixa prevalência e, assim, contribui para a subnotificação dos casos. Estudos que analisaram a influência da ampliação do número de lâminas de Kato-Katz examinadas, novos métodos ou a combinação de vários métodos de diagnóstico verificaram que 0 Kato-Katz subnotificava a prevalência da doença quando comparado aos demais métodos, apesar de a ampliação no número de lâminas examinadas aumentar sua sensibilidade., ${ }^{9,10,19-22}$

Observa-se, outrossim, que as equipes do PCE realizam os inquéritos nas mesmas áreas, sucessivas vezes, por razões técnicas e operacionais. ${ }^{23-25}$ No presente estudo, o município foi amostrado de acordo com sua composição populacional urbana e rural, tendo-se levado em consideração o número de UBS e a população atendida por cada uma delas. Para a maioria das localidades, a positividade encontrada neste estudo foi superior à reportada pelo inquérito do PCE local. 0 emprego de um inquérito representativo da população do município, associado à redução no número de indivíduos participantes e ao aumento no número de lâminas analisadas para cada um deles, mostrou-se mais efetivo em revelar a prevalência da esquistossomose nas localidades estudadas.

Diante do cenário descrito, é possível pensar em algumas estratégias de otimização dos trabalhos do PCE no âmbito municipal, como as listadas a seguir.

1) Integração às ações dos ACS da função de orientar e/ou coletar o material para exame coproparasitológico em sua área de abrangência, conforme recomendação do Ministério da Saúde e comprovação acadêmica de sua eficácia. ${ }^{25-27}$ Essa estratégia foi pensada devido ao modelo de trabalho adotado pelo município do estudo, onde os ACSs são agentes ativos e atuam de forma a promover 0 acesso dos indivíduos aos serviços assistenciais, diagnóstico precoce e tratamento oportuno.

2) Seleção aleatória, porém de forma representativa, dos indivíduos componentes da amostra do inquérito, ano a ano, com vistas a se conhecer a positividade real 
das localidades de Lagoa da Canoa, para o agravo e 0 cenário epidemiológico do município, e não de locais restritos. A medida é igualmente eficiente no controle da morbidade, redução da prevalência, avaliação e monitoramento das ações.

3) Redução do número de indivíduos participantes e ampliação do número de lâminas examinadas para cada indivíduo. A estratégia mostra-se necessária pela própria racionalidade, uma vez que a média de lâminas lidas, no município analisado, é de 2.000 anuais, o que representa 2.000 pessoas. Neste estudo, foram lidas 2.088 lâminas de 348 indivíduos, sendo encontrada uma diferença de pelo menos duas vezes na positividade dos indivíduos avaliados, comparada à positividade dos indivíduos-objeto dos inquéritos do PCE local.

Sem correção nas positividades, apenas cinco localidades teriam indivíduos positivos e conviventes sendo tratados, e três localidades com tratamento coletivo. Mediante a correção das positividades, foram encontrados indivíduos positivos e conviventes sendo tratados em 12 localidades, e em 13, tratamento coletivo. Este dado é muito importante: mostra que, mesmo sem um inquérito representativo e com ampliação no número de lâminas por indivíduo, medidas propostas neste estudo, as categorias de tratamento preconizadas pelo Ministério da Saúde não se alinham mais à

\section{Referências}

1. World Health Organization - WHO. Schistosomiasis WHO guideline development group proposal guidelines for implementation of control and elimination of schistosomiasis and verification of interruption of transmission [Internet]. Geneva: WHO; 2020 [cited 2020 Dec 18]. Available from: https:/www.who.int/schistosomiasis/news/publicconsultation-experts-guideline-development-group/ en/

2. Neiva AH. Aspectos geográficos da imigração e colonização do Brasil. Rev Bras Geog. 1947;9:249-70.

3. Ministério da Saúde (BR). Secretaria de Vigilância em Saúde. Coordenação-Geral de Desenvolvimento da Epidemiologia em Serviços. Guia de vigilância em saúde: volume único [Internet]. 3. ed. Brasília: Ministério da Saúde; 2019 [citado 2020 dez 18]. Disponível em: https://portalarquivos2.saude.gov.br/ images/pdf/2019/junho/25/guia-vigilancia-saudevolume-unico-3ed.pdf realidade epidemiológica do país, sobretudo devido ao modelo de análise parasitológica preconizado pelo Ministério da Saúde e praticado pelos técnicos do PCE.

Em seu conjunto, os dados do estudo mostram que a coleta de três amostras de material fecal e 0 emprego da leitura de seis lâminas de Kato-Katz aumentam a positividade para a esquistossomose em área de baixa endemicidade. Como conclusão, fica evidenciado que a esquistossomose mansoni continua a ser um agravo de grande magnitude para os municípios da área endêmica e uma prioridade a incluir nas agendas de políticas de promoção da saúde no Brasil.

\section{Contribuição dos autores}

Santos IGA delineou a pesquisa, coletou, analisou e interpretou os dados, redigiu e revisou o manuscrito. Santos Júnior EG, Alves LC e Brayner AF analisaram e interpretaram os dados, e revisaram o manuscrito. Bezerra LP, Cirilo TM, Silva LO, Machado JPV, Lima PD, Souza MRB, Gomes SC, Silva GIL, Damasceno IA, Alencar VJB, Carvalho MMV, Ramos RES, Gomes DS e Paz WS coletaram e analisaram os dados, e revisarem 0 manuscrito. Todos os autores aprovaram a versão final do manuscrito, garantindo sua precisão e integridade.

4. Rocha TJM, Santos MCS, Lima MVM, Calheiros CML, Wanderley FS. Aspectos epidemiológicos e distribuição dos casos de infecção pelo Schistosoma mansoni em municípios do Estado de Alagoas, Brasil. Rev Pan-Amaz Saúde [Internet]. 2016 jun [citado $2020 \mathrm{dez} 18$ ];7(2):27-32. https://doi.org/doi. org/10.5123/S2176-62232016000200003

5. Palasio RGS, Bortoleto NA, Rosa-Xavier IG, Andrighetti MTM, Tuan R, Chiaravalloti-Neto F. Schistosomiasis in the Middle Paranapanema river region, state of São Paulo, Brazil: does it matter today for public health? Rev Soc Bras Med Trop [Internet]. 2019 Jun [cited 2020 Dec 18];52:e20180447. Available from: https:// doi.org/doi.org/10.1590/0037-8682-0447-2018

6. Katz N, Chaves A, Pellegrino J. A simple device for quantitative stool thick smear technique in Schistosoma mansoni. Rev Inst Med Trop São Paulo [Internet]. 1972 Nov-Dec [cited 2020 Dec 18];14(6):397-400. Available from: http://www.imt. usp.br/wp-content/uploads/revista/vol14/397-400.pdf 
7. World Health Organization - WHO. Prevention and control of schistosomiasis and soil-transmitted helminthiasis: report of a WHO expert committee [Internet]. Geneva: WHO; 2002 [cited 2020 Dec 18]. Available from: https://apps.who.int/iris/ bitstream/handle/10665/42588/WHO_TRS_912. pdf?sequence $=1 \&$ isAllowed $=\mathrm{y}$

8. Pinheiro MCC, Carneiro TR, Hanemann ALP, Oliveira SM, Bezerra FSM. The combination of three faecal parasitological methods to improve the diagnosis of schistosomiasis mansoni in a low endemic setting in the state of Ceará, Brazil. Mem Inst Oswaldo Cruz [Internet]. 2012 Nov [cited 2020 Dec 18];107(7):873-6. Available from: https://doi. org/10.1590/s0074-02762012000700006

9. Lamberton PH, Kabatereine NB, Oguttu DW, Fenwick A, Webster JP. Sensitivity and specificity of multiple Kato-Katz thick smears and a circulating cathodic antigen test for Schistosoma mansoni diagnosis pre- and post-repeated-praziquantel treatment. PLoS Negl Trop Dis [Internet]. 2014 Set [cited 2020 Dec 18];8(9):e3139. Available from: https://doi. org/10.1371/journal.pntd.0003139

10. Bezerra FSM, Leal JKF, Sousa MS, Pinheiro MCC, Ramos Júnior AN, Silva-Moraes V, et al. Evaluating a point-of-care circulating cathodic antigen test (POCCCA) to detect Schistosoma mansoni infections in a low endemic area in north-eastern Brazil. Acta Trop [Internet]. 2018 Jun [cited 2020 Dec 18];182:26470. Available from: https://doi.org/10.1016/j. actatropica.2018.03.002

11. World Health Organization - WHO. Enhancing implementation of schistosomiasis control and elimination programmes [Internet]. Geneva: WHO; 2020 [cited 2020 Dec 18]. Available from: https:// www.who.int/activities/enhancing-implementation-ofschistosomiasis-control-and-elimination-programmes

12. Governo do Estado de Alagoas (BR). Secretaria de Estado do Planejamento, Gestão e Patrimônio. Perfil municipal: Lagoa da Canoa [Internet]. Maceió: Secretaria de Estado do Planejamento, Gestão e Patrimônio; 2018 [citado $2020 \mathrm{dez} 18$ ]. 35 p. Disponível em: http://dados.al.gov.br

13. Governo do Estado de Alagoas (BR). Secretaria de Estado da Saúde de Alagoas. Superintendência de Vigilância em Saúde. Gerência de Informação e Análise da Situação de Saúde. Coordenação Técnica, Produção e Organização. Saúde Alagoas: análise da situação de saúde 2017. Livro $7^{\text {a }}$ Região de Saúde [Internet]. Maceió: Secretaria de Estado da Saúde de Alagoas; 2017 [citado $2020 \mathrm{dez}$ 18]. 127 p. Disponível em: https://www.saude.al.gov.br
14. Barbosa CS, Gomes ECS, Marcelino JMR, Cavalcante KRLJ, Nascimento WRC. Quality control of the slides by Kato-Katz method for the parasitological diagnosis of schistosomiasis infection by Schistosoma mansoni. J Bras Patol Med Lab [Internet] 2017 [cited 2020 Dec 18];53(2):110-4. Available from: https:// doi.org/10.5935/1676-2444.20170018

15. Landis JR, Koch GG. The measurement of observer agreement for categorical data. Biometrics [Internet]. 1977 Mar [cited 2020 Dec 18];33(1):159-74. Available from: https://doi.org/10.2307/2529310

16. Ministério da Saúde (BR). Secretaria de Vigilância em Saúde. Departamento de Vigilância em Doenças Transmissíveis. Plano integrado de ações estratégicas de eliminação da hanseníase, filariose, esquistossomose e oncocercose como problema de saúde pública, tracoma como causa de cegueira e controle das geohelmintíases: plano de ação 20112015 [Internet]. Brasília: Ministério da Saúde; 2012 [citado 2020 dez 18]. Disponível em: http://bvsms. saude.gov.br/bvs/publicacoes/plano_integrado_ acoes_estrategicas_2011_2015.pdf

17. Ministério da Saúde (BR). Secretaria de Vigilância em Saúde. Departamento de Vigilância das Doenças Transmissíveis. Vigilância da esquistossomose mansoni: diretrizes técnicas [Internet]. 4. ed. Brasília: Ministério da Saúde; 2014 [citado 2020 dez 18]. 144 p. Disponível em: http://bvsms.saude.gov.br/ bvs/publicacoes/vigilancia_esquistossome_mansoni_ diretrizes_tecnicas.pdf

18. Katz N. Inquérito nacional de prevalência da esquistossomose mansoni e geo-helmintoses [Internet]. Belo Horizonte: CPqRR; 2018 [citado 2020 dez 18]. 76 p. Disponível em: https://www.arca. fiocruz.br/handle/icict/25662

19. Sousa SEM, Carvalho AQ, Cardoso JFN, Coelho PMZ, Geiger SM, Enk MJ. Schistosomiasis in the Amazon region: is the current diagnostic strategy still appropriate?. Rev Soc Bras Med Trop [Internet]. 2017 Dec [cited 2020 Dec 18];50(6):848-52. Available from: https://doi.org/10.1590/0037-8682-0097-2017

20. Oliveira WJ, Magalhães FDC, Elias AMS, Castro VN, Favero V, Lindholz CG, et al. Evaluation of diagnostic methods for the detection of intestinal schistosomiasis in endemic areas with low parasite loads: saline gradient, Helmintex, Kato-Katz and rapid urine test. PLoS Negl Trop Dis [Internet]. 2018 Feb [cited 2020 Dec 18];12(2):e0006232. Available from: https://doi. org/10.1371/journal. pntd.0006232 
21. Okoyo C, Simiyu E, Njenga SM, Mwandawiro C. Comparing the performance of circulating cathodic antigen and Kato-Katz techniques in evaluating Schistosoma mansoni infection in areas with low prevalence in selected counties of Kenya: a crosssectional study. BMC Public Health [Internet]. 2018 Apr [cited 2020 Dec 18];18(1):478. Available from: https://doi.org/10.1186/s12889-018-5414-9

22. Sousa MS, van Dam GJ, Pinheiro MCC, Dood CJ, Peralta JM, Peralta RHS, et al. Performance of an ultra-sensitive assay targeting the circulating anodic antigen (CAA) for Detection of Schistosoma mansoni Infection in a low endemic area in Brazil. Front Immunol [Internet]. 2019 Apr [cited 2020 Dec 18];10:682. Available from: https://doi. org/10.3389/fimmu.2019.00682

23. Farias LMM, Resendes APC, Sabroza PC, SouzaSantos R. Análise preliminar do Sistema de Informação do Programa de Controle da Esquistossomose no período de 1999 a 2003. Cad Saúde Pública [Internet]. 2007 jan [citado 2020 dez 18];23(1):235-9. Disponível em: https://doi. org/10.1590/S0102-311X2007000100025

24. Quinino LRM, Costa JMBS, Aguiar LR, Wanderley TNG, Barbosa CS. Avaliação das atividades de rotina do Programa de Controle da Esquistossomose em municípios da Região Metropolitana do Recife,
Pernambuco, entre 2003 e 2005. Epidemiol Serv Saúde [Internet]. $2009 \mathrm{dez}$ [citado $2020 \mathrm{dez}$ 18];18(4):335-43. Disponível em: https://doi. org/10.5123/S1679-49742009000400003

25. Costa CS, Rocha AM, Silva GS, Jesus RPFS, Albuquerque AC. Programa de controle da esquistossomose: avaliação da implantação em três municípios da Zona da Mata de Pernambuco, Brasil. Saúde Debate [Internet]. 2017 mar [citado 2020 dez 18];41(esp):229-41. Disponível em: https://doi. org/10.1590/0103-11042017s17

26. Ministério da Saúde (BR). Secretaria de Atenção à Saúde. Departamento de Atenção Básica. Política nacional de atenção básica [Internet]. Brasília: Ministério da Saúde; 2012 [citado 2020 dez 18]. Disponível em: http://189.28.128.100/dab/docs/ publicacoes/geral/pnab.pdf

27. Cesarino MB, Dido MR, Ianni AMZ, Vicentini ME, Ferraz AA, Chiaravalloti-Neto F. A difícil interface controle de vetores - atenção básica: inserção dos agentes de controle de vetores da dengue junto às equipes de saúde das unidades básicas no município de São José do Rio Preto, SP. Saúde Soc [Internet]. 2014 set [citado 2020 dez 18];23(3):1018-32. Disponível em: https://doi.org/10.1590/S010412902014000300023 


\begin{abstract}
Objective:To analyze aspects related to schistosomiasis positivity in an area oflow prevalence in Brazil. Methods: This was a cross-sectional study, carried out in the first half of 2020, where we analyzed the proportion of positivity, according to the number of Kato-Katz slides, the diagnostic performance of the test and positivity estimates based on data from the Schistosomiasis Surveillance and Control Program Information System (SISPCE). Results: 2,088 slides from 348 individuals were analyzed, with proportion of positivity of $11.8 \%$, $26.7 \%$ and $31.0 \%$ for 1,4 and 6 slides analyzed, respectively. There was excellent agreement (Kappa $=$ $0.91)$ between the readings of 4 and 6 slides. The SISPCE data was estimated to be underreported by up to 2.1 times. Conclusion: Increasing the number of slides increased Kato-Katz positivity, which can contribute to maximizing the control of the disease as a Public Health problem.
\end{abstract}

Keywords: Neglected Diseases; Cross-Sectional Studies; Sensitivity and Specificity; Public Health; Schistosomiasis Mansoni; Parasitic Diseases.

\section{Resumen}

Objetivo: Analizar aspectos relacionados con la positividad para esquistosomiasis en área de baja prevalencia en Brasil. Métodos: Estudio transversal, realizado en el primer semestre de 2020, donde se analizó la proporción de positividad según el número de portaobjetos de Kato-Katz, el rendimiento diagnóstico de la prueba y la creación de un factor de estimación de positividad a partir de los datos del Sistema de Información del Programa de Vigilancia y Control de la Esquistosomiasis (SISPCE). Resultados: Se analizaron 2.088 láminas de 348 individuos, con proporción de positividad del 11,8\%, 26,7\% y 31,0\% para 1, 4 y 6 láminas analizadas, respectivamente. Hubo una excelente concordancia $($ Kappa $=0,91)$ en la comparación entre la lectura de 4 y 6 láminas. Se estimó un subregistro de 2,1 veces en los datos del SISPCE. Conclusión:Aumentar el número de muestras aumentó la positividad de Kato-Katz, lo que puede contribuir a maximizar el control de la enfermedad como problema de Salud Pública.

Palabras clave: Enfermedades Desatendidas; Estudios Transversales; Sensibilidad y Especificidad; Salud Pública; Esquistosomiasis Mansoni; Enfermedades Parasitarias.

Recebido em 04/07/2020

Aprovado em 31/10/2020

Editora associada: Bárbara Reis-Santos - — o orcid.org/0000-0001-6952-0352
Editora científica:Taís Freire Galvão - @ orcid.org/0000-0003-2072-4834 Editora geral: Leila Posenato Garcia - @ orcid.org/0000-0003-1146-2641 